\title{
A signal detection theory analysis of a category judgment experiment'
}

\author{
ELIZABETH F. SHIPLEY ${ }^{2}$ \\ UNIVERSITY OF PENNSYL VANIA AND EASTERN PENNSYL VANIA PSYCHIATRIC INSTITUTE
}

A category-judgment experiment is reported. Rating-scale judgments of loudness were made with five or eight intensity levels of a tone and with different frequencies of presentation of the stimuli. Obtained ROC curves for pairs of stimuli were judged io support application of signal-detection theory. However, certain common assumptions of the theory are called into question by the data. Discrimination was poorer when more categories were available. This is attributed to an increase in criterion variance with an increase in the number of criteria. The slope of the ROC curves for different stimulus pairs suggested that stimulus variance is neither constant nor does it necessarily increase with stimulus intensity. It is argued that stimulus variance may depend upon the distribution of internal observations over all trials.

According to the theory of signal detection (Green \& Swets, 1966), performance in any experimental condition is considered to be a function of two kinds of effects: (a) stimulus or sensory effects that hopefully are invariant for stimulus presentations with the same physical properties, and (b) response or decision effects that reflect the $O$ 's varying approach to the task. In this paper we are asking, within the framework of signal-detection theory (SDT), if stimulus effects are indeed the same with certain variations in the procedure. But we are also asking a methodological question, namely, within what limits can one determine stimulus effects independently of response effects? This is a report of an experimental study in which we varied: (a) the number of responses available to the $O,(b)$ the frequency of presentation of different stimuli within a block of trials, and (c) the number of different intensity levels. We will examine the obtained ROC curves for answers to these questions.

The ROC curve is a way of relating performance with two different stimulus values when all else is constant. It is a plot of the relative frequency of a specific response on trials with a specific stimulus value against the relative frequency of the same response on trials with a different stimulus value. In practice, holding "all else constant" is accomplished by presenting the two stimulus values within the same block of trials. The best known ROC curve is that for yes-no detection experiments. The response is judging "Yes, a signal was presented," and the two stimulus conditions are the presentation of a signal in a noise background and the presentation of noise alone. In this case, the ROC curve is the probability of responding "Yes" when a signal was presented plotted against the probability of responding "Yes" when a signal was not presented.

Usually ROC curves are plotted for procedures in which only two stimulus values were used, e.g., signal and no-signal. In such cases, every trial is counted in determining a data point. In such category-judgment procedures as the one reported here, more than two different stimulus intensities are presented within a block of trials. However, only the trials with one of two specific stimulus values are examined to obtain data points for a specific ROC curve. Of course, ROC curves can be obtained for each pair of stimuli so that data points for many different ROC curves can be obtained from a single block of trials. We will consider how one gets more than one point for a specific ROC curve after we consider some of the assumptions of SDT and a formula for the ROC curve.

The version of SDT considered here appears to be the one that has had the greatest success in interrelating the results from different experimental situations (Green \& Swets, 1966). Obviously, many aspects of SDT are omitted from this outline-omissions that hopefully are irrelevant to the issues considered here.

We are assuming that each presentation of a stimulus yields an internal observation. For repeated presentations of the same stimulus, the internal observations are normally distributed. When two stimuli vary on a single physical dimension (e.g., intensity), the internal distributions corresponding to the two stimuli are on the same continuum. However, internal distributions corresponding to different stimuli may differ in variance. In order to respond, the $O$ sets a criterion or criteria on the internal continuum. These criteria may vary in location from moment to moment. If a criterion does vary in location, the distribution of these locations is also normal. On each trial, the relations between the internal observation and the criteria determine the $O$ 's response-for instance, he judges "Loud" if the observation exceeds a certain criterion. ${ }^{3}$

If the assumptions of SDT outlined above are valid, and if criterion variance is constant, then the ROC curve is a straight line on normal-normal coordinates. Specifically, consider two stimuli, $A$ and B, with internal distributions $N\left(\mu_{A}, \sigma_{A}\right)$ and $\mathrm{N}\left(\mu_{\mathrm{B}}, \sigma_{\mathrm{B}}\right)$. Let $\sigma_{\mathrm{K}}$ represent the standard deviation of the criterion distributions. Let $z\left(R_{x} \mid B\right)$ represent the $z$ transform of the probability of response $R_{x}$ given Stimulus $B$. The response $R_{X}$ indicates an observation greater than or equal to the criterion $x$. With these conditions, the equation for the ROC curve on normal-normal coordinates is

$$
\begin{aligned}
z\left(R_{x} \mid B\right)= & z\left(R_{x} \mid A\right)\left[\frac{\left(\sigma_{A}^{2}+\sigma_{\mathrm{K}}^{2}\right)^{1 / 2}}{\left(\sigma_{\mathrm{B}}^{2}+\sigma_{\mathrm{K}}^{2}\right)^{1 / 2}}\right] \\
& +\left[\frac{\mu_{\mathrm{B}}-\mu_{\mathrm{A}}}{\left(\sigma_{\mathrm{B}}^{2}+\sigma_{\mathrm{K}}^{2}\right)^{1 / 2}}\right]
\end{aligned}
$$

Note that we have assumed criterion variance, $\sigma^{2}{ }_{K}$, to be constant. The usual assumption is to take criterion variance to be negligible. With this latter assumption, the slope of the ROC curve is completely determined by the ratio $\sigma_{\mathrm{A}} / \sigma_{\mathrm{B}}$. Empirical ROC curves frequently have a slope less than unity. This has been explained by assuming that stimulus variability increases with stimulus intensity ${ }^{4}$ or, alternatively, by abandoning the Gaussian assumption and assuming the stimulus distributions to be Rayleigh or exponential (Green \& Swets, 1966, Chap. 3 and Chap. 6). However, as Wickelgren (1968) has pointed out, if criterion variance, $\sigma^{2} \mathrm{~K}$, is sizable, then the slope of the ROC curve is not equal to the ratio $\sigma_{A} / \sigma_{B}$. Moreover, when $\sigma_{K}$ is large relative to $\sigma_{A}$ and $\sigma_{B}$, the slope will be near unity even if $\sigma_{A}$ and $\sigma_{B}$ differ greatly.

Furthermore, the various measures of discriminability (the distance from the origin to some point on the ROC curve; Green \& Swets, 1966, pp. 96-98) also depend upon criterion variance, $\sigma^{2} \mathbf{K}$. As we can see in Eq. 1, with an increase in criterion variance, the various measures of discriminability decrease. 
In practice, data points for ROC curves are obtained in two different ways. In binary procedures, the $\mathbf{O}$ uses two possible responses and is assumed to set a single criterion. A block of trials yields a single data point. By inducing the $O$ to change the location of the criterion, different points are obtained. In rating-scale procedures, the $O$ uses more than two responses, responses that are ordered on a psychological dimension, e.g., "Loud," "Medium," and "Soft." Criteria are assumed to be set that divide the continuum into segments corresponding to the different responses. With this procedure, a block of trials yields as many data points as there are criteria. All responses above a criterion are combined for an estimate of the coordinates of a single point (Green \& Swets, 1966,pp. 99-103).

Nachmias (1968) and Wickelgren (1968) have suggested that when the $O$ works with several criteria, the variance of each criterion may be greater than when the $O$ has but a single criterion. If this is so, discriminability will be poorer than with a binary procedure. It is not clear that empirical ROC curves from rating-scale procedures and from binary-choice procedures are equivalent. Some evidence exists that they are: Egan, Schulman, and Greenberg (1959), Swets, Tanner, and Birdsall (1961, footnote), Emmerich (1968, Ss 2 and 4), and Nachmias (1968). However, evidence also exists that rating scales yield a lower measure of stimulus discriminability: Swets, Tanner, and Birdsall (1961), and Emmerich (1968, $\mathrm{S} 3$ ). Although it is certainly more efficient to generate ROC curves with rating scales, such curves are of limited interest if they vary with the number of responses permitted. In this experiment, we compare ROC curves when three, five, or nine responses are permitted.

Are ROC curves for a specific pair of stimuli independent of the other stimuli presented within the same series of trials? In terms of SDT, the question becomes: Are the internal distributions for Stimulus $A$ and for Stimulus B independent of the other stimulus values presented within a block of trials? Under some detection conditions, they evidently are not. When signals are used that differ qualitatively (e.g., in pitch) from trial to trial, detection performance is poorer than when the signal has the same frequency every time it is presented (Green \& Swets, 1966, pp. 283-289). Detection performance has also been studied with signals that differ in intensity from trial to trial. Emmerich (1968) studied yes-no detection performance. He reports the same ROC curve whether or not a second signal of a different intensity is presented on some trials within a block.

On the other hand, the relative frequency of presentation of the signal sometimes makes a difference. Markowitz and Swets (1967), who found that rating scales and binary techniques yield ROC curves with different slopes, attribute these differences to variation in the a priori probability of the signal in the binary-choice task. ${ }^{5}$ With an increase in the frequency of presentation of the signal, the $O$ 's "knowledge of the stimulus"-which is perhaps knowledge of what to attend to-is thought to increase with a concomitant increase in sensitivity to that specific stimulus. (See also Swets \& Sewall, 1961, for further discussion and references.)

In the study reported here, we are also concerned with the effects of other stimuli upon the ROC curve. We elected to work with clearly audible stimuli that vary on a single dimension. We varied the relative frequency of stronger and weaker stimuli. The $O$ rated the stimuli for apparent loudness. In such a task, the $\mathrm{O}$ has no difficulty in deciding what to listen for-he cannot fail to hear the stimulus - and, hence, the effect of "knowledge of the stimulus" should be slight.

\section{METHOD \\ Observers}

Two undergraduates at the University of Pennsylvania served as Os. They were hired through the student employment of fice and paid an hourly wage. Neither reported any known hearing defects.

\section{Apparatus}

The stimulus was a $1,000-\mathrm{Hz}$ tone of 143 msec duration, generated by a Kronhite pushbutton oscillator. It was gated by a sign-zero switch, passed through an alternator and a variable-resistance network controlled by a tape reader, and then presented binaurally through PDR- 8 earphones to the $O$ in a sound-insulated room. The $O$ had a response box with nine keys in a line. The ends of the box were labeled "Soft" and "Loud." On a given day, either nine keys or the five middle keys or the three middle keys were exposed.Stimuli and responses were recorded on a pen recorder. A trial lasted $2.5 \mathrm{sec}$.

\section{Design}

Nine experimental conditions were used with each $\mathrm{O}$; these consisted of all possible combinations of the three stimulus conditions and the three response conditions.

Stimulus conditions: (a) five stimulus levels, (b) five stimulus levels with biased frequency of presentation (a preponderance of soft stimuli), and (c) eight stimulus levels. Five stimulus intensities were common to all conditions; they are referred to as $A$ through $E$ in order of increasing intensity. The three additional intensities used in the

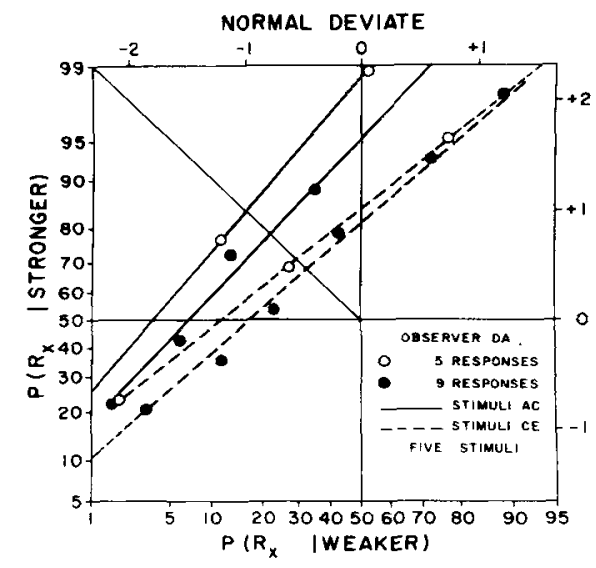

Fig. 1. ROC curves obtained with rating scales plotted on normal-normal coordinates. Open circles indicate a five-response scale; filled circles indicate a nine-response scale. Five stimulus levels were presented within a block of trials: $A$ is the weakest intensity; $C$, the middle intensity; and $E$, the strongest intensity. Data are given for two stimulus pairs from each condition. Response proportions for the stronger intensity of a pair are always plotted on the ordinate. Straight lines were fit by eye.

eight-stimuli condition were intermediate between $A$ and $B$, between $B$ and $C$, and between $D$ and $E$. In the five-stimuli condition, the most intense stimulus, E, was presented slightly more often (on approximately $29 \%$ of the trials) than the other stimuli, which occurred equally of ten. In the eight-stimuli condition, both $D$ and $E$ were presented slightly more often (on approximately $20 \%$ and $18 \%$ of the trials, respectively). In the biased-frequency condition, the least intense stimulus, $A$, was presented on approximately $40 \%$ of the

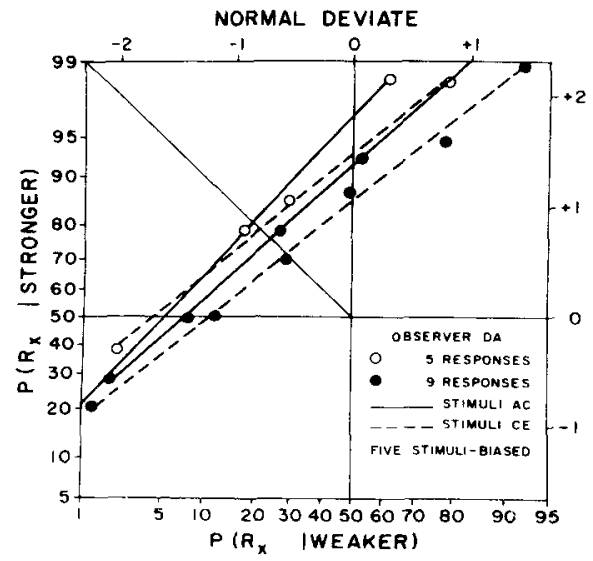

Fig. 2. Same as Fig. 1 except that Stimulus A was presented relatively more often. 


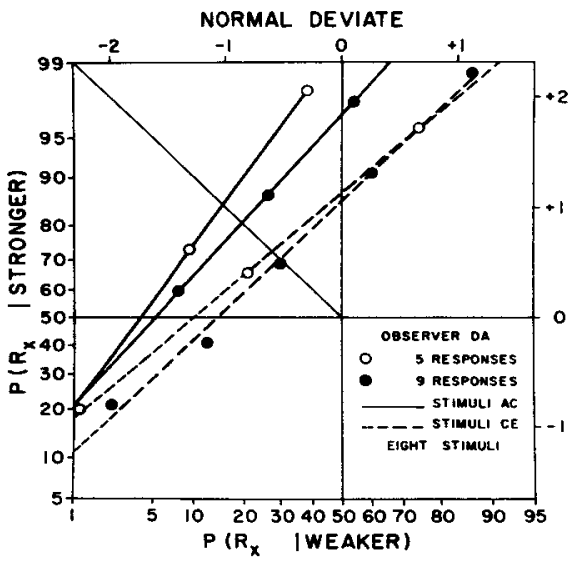

Fig. 3. Same as Fig. 1 except that eight stimulus levels were used: three levels were between $A$ and $C$ in intensity, two levels were between $C$ and $E$ in intensity.

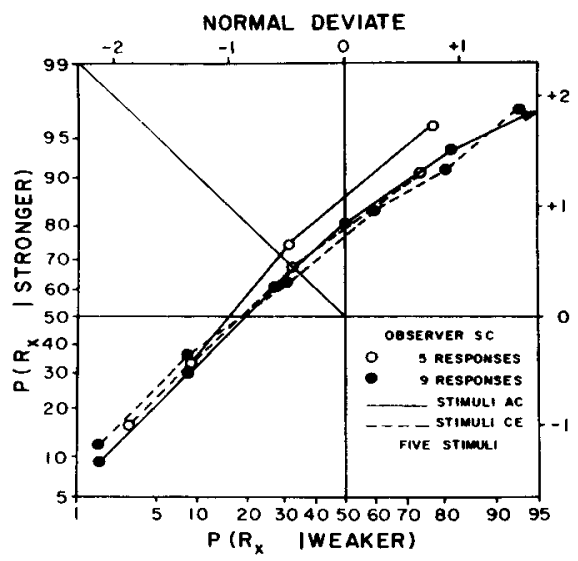

Fig. 4. Same as Fig. 1 except that adjacent data points from the same condition and the same stimulus pair are connected by straight lines.

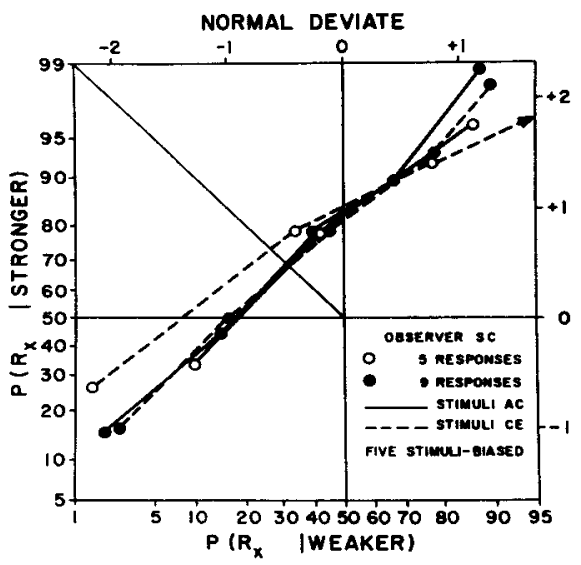

Fig. 5. Same as Fig. 2 except that adjacent points from the same condition and the same stimulus pair are connected by straight lines. trials. Other stimuli occurred equally often. The stimuli were clearly audible. No background noise or tone was presented. Voltage was measured across the earphones. For Stimuli A through E, respectively, the values were $-26.7,-24.9,-23.0,-21.5$, and $-20.4 \mathrm{~dB}$ re $1 . \mathrm{V}$ rms.

Response conditions: (a) Three different responses, (b) five different responses, or (c) nine different responses were permitted.

\section{Procedure}

Each $O$ was run separately for 16 days, with a 2 -h session each day. The first 2 days were considered practice days, and data from these days are not included in the analysis. Within a session, two conditions were run; the same response condition was used with two different stimulus conditions. Each day, two blocks of 500 trials were run on each of the two conditions, for a total of 2,000 trials per session. The Os were instructed to respond on the basis of "how loud the tone sounds." They were told there were no right answers and that their task was to indicate apparent loudness by selecting a key. The Os were given no further instruction on the use of the keys, and they were never told the composition of the stimulus sets. There was no feedback.

\section{RESULTS AND DISCUSSION}

We will confine our analysis to SDT, and within this framework, we will examine the shape of the ROC curve and the value of a discriminability index, $\mathbf{d}_{\mathrm{e}}^{\prime}$, for certain pairs of stimuli.

Several discriminability measures can be obtained from ROC curves, measures that differ in value when the slope of the curve is not unity on normal-normal coordinates (see Green \& Swets, 1966, pp. 96-99). The value of $d_{e}^{\prime}$ is twice the absolute value of the normal deviate at the intersection of the ROC curve and the negative diagonal. We have used $d_{e}^{\prime}$ because it reflects the variance of both stimulus distributions.

First we ask if the data warrants a SDT analysis. Data points for the Stimulus Pair $A$ and $\mathrm{C}$ and for the Stimulus Pair $\mathrm{C}$ and $\mathrm{E}$ are given in Figs. 1-7 on normal-normal coordinates. For O DA, straight lines have been fitted by eye to the data points. There appears to be no consistent deviation from linearity (Figs. 1, 2, and 3). For O SC, straight lines do not provide as good a fit. In Figs. 4, 5, and 6, adjacent data points have been connected by straight lines to make more apparent systematic deviations from linearity-if such exist. If anything, one might judge some curves ( 6 of 12 to my eye) to be concave downward. This is the usual finding when empirical ROC curves systematically deviate from linearity. Wickelgren (1968) attributed concave downward ROC curves to greater variance for the extreme criteria. ${ }^{6}$

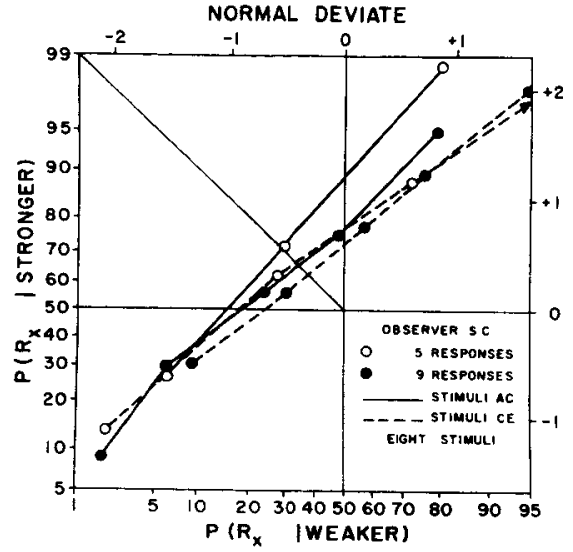

Fig. 6. Same as Fig. 3 except that adjacent data points from the same condition and the same stimulus pair are connected by straight lines.

The failure to find a concave downward trend in the data of ODA, and its sporadic appearance in the data of SC, suggests that the phenomenon should either be ignored or attributed to criterion variability. For the purposes of this discussion, we will consider the empirical ROC curves for both Os to be linear and, hence, to support the SDT position. Straight lines have been fitted to each set of data. Estimates of $d_{e}^{\prime}$ and the slope of these curves are given in Tables 1 and 2.

Three aspects of the data will be examined: (a) the effect of the number of response alternatives, i.e., the number of categories in the rating scale; (b) the dependence of the slope of the ROC curve upon the specific stimulus values and the stimulus conditions; and (c) the dependence of the frequency of use of different responses upon the stimulus conditions and the response conditions. The first two

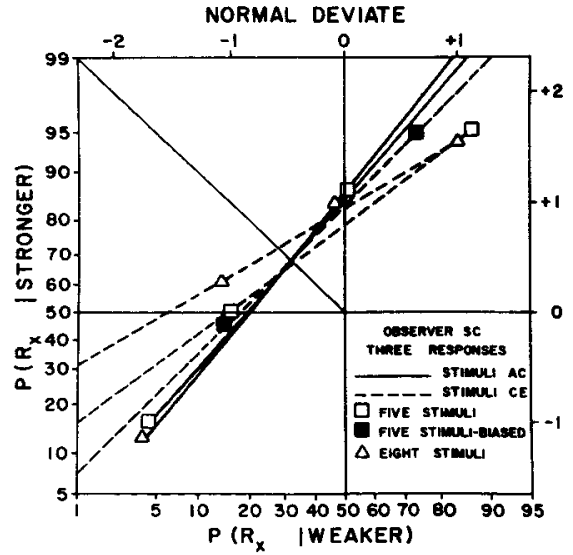

Fig. 7. ROC curves for the three-category scale from the three stimulus conditions. Data are presented only if two data points were obtained in the range of response proportions from .01 to .99 . 
Table 1

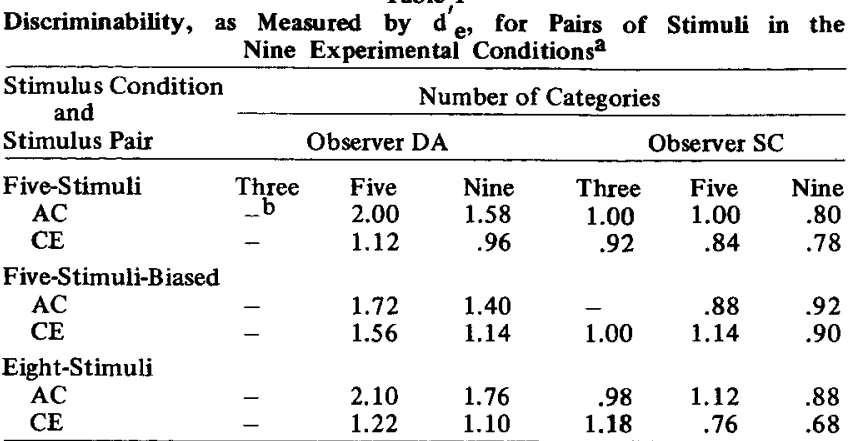

See text for the definition of $d^{\prime}$. Curves were fit to the data by eye. $b \quad R O C$ curves were based on data points with response proportions between .01 and .99. Dashes indicate a failure to obtain a minimum of two data points in this range.

aspects of the data will raise questions about the assumptions of SDT. Let us consider these three aspects in order.

We can see in Figs. 1-6 that discriminability is better when five responses are used than when nine responses are used. The data for O SC in Fig. 5, Stimuli $A$ and $C$, provide the single exception. The estimates of $\mathrm{d}_{\mathrm{e}}^{\prime}$ support this conclusion (Table 1). When one considers adjacent pairs of stimuli (e.g., A and B, B and C, etc.), discriminability is also better ( $\mathrm{d}_{\mathrm{e}}^{\prime}$ is larger) for five responses than for nine responses for O DA (in 11 of 12 comparisons) and for O SC ( 10 of 12 comparisons).

What of the data from the three-category conditions? For ODA, only three ROC curves could be plotted and these were for adjacent pairs of stimuli. (That is, in only three instances were two data points found within the square bounded by .01 and .99.) For these cases, $d_{e}^{\prime}$ was clearly larger when three responses were permitted. For O SC, there was no consistent difference in the values of $d_{e}^{\prime}$ obtained with three categories and those obtained with five categories (Table 1).

In sum, an increase in the number of possible responses leads at some point to a decrease in apparent discriminability. This is to be expected if criterion variance increases as the number of criteria increases.

As we noted above, theoretically the slope of the ROC curve approaches unity as criterion variance, $\sigma^{2} \mathrm{~K}$, increases (Eq. 1). If poorer discrimination with nine categories is due to an increase in criterion variance, then the slope of the various ROC curves obtained with nine categories should be closer to unity than the slopes of the corresponding curves obtained with five categories. Insofar as differences in slopes are apparent, the differences are consistent with this hypothesis. Five of the six comparisons for each $\mathrm{O}$ support this hypothesis (Table 2). Thus, the slope of the obtained ROC curves as well as the discriminability index, $d_{\mathrm{e}}^{\prime}$, suggest that criterion variance increases as the number of criteria increases.

The slope of the ROC curve is consistently different for different stimulus pairs. This can be seen when the curves relating performance with the softest tone, $\mathrm{A}$, and the middle tone, $\mathrm{C}$, are compared to curves relating performance with the middle tone, $\mathrm{C}$, and the loudest tone, E (Figs. 1-7). ROC curves for the less intense pair of stimuli are always steeper (Table 2). In fact, contrary to all previous findings, the slope of the curve for Stimuli $A$ and $C$ tends to be greater than unity in both the five-stimuli condition and the eight-stimuli condition. Curves for Stimuli $\mathrm{C}$ and $\mathrm{E}$, on the other hand, all have a slope of unity or less in all conditions (Table 2).

Table 2

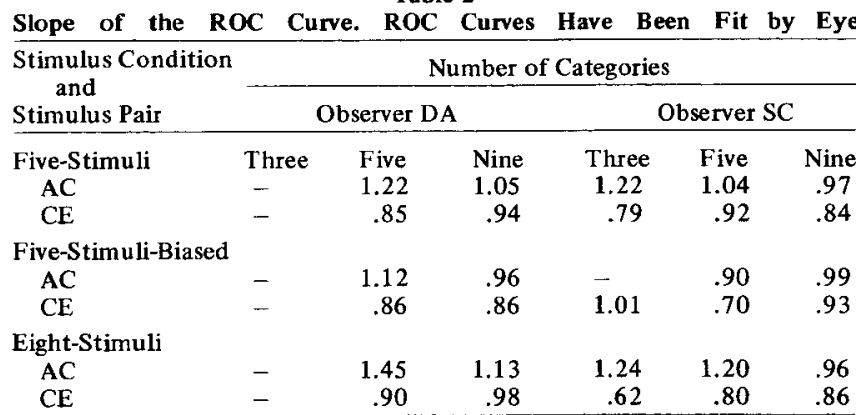

Let us consider the five-stimuli condition and the eight-stimuli condition further. If we take the slope of the ROC curve to indicate the ratio of the standard deviations of the stimulus distributions, then $\sigma_{\mathrm{C}}$ must be less than $\sigma_{A}$. Since the curves for Stimuli $\mathrm{C}$ and $\mathrm{E}$ have slopes less than unity, $\sigma_{\mathrm{C}}$ must also be less than $\sigma_{\mathrm{E}}$. Thus, these results suggest that stimulus variance is least for a stimulus of intermediate intensity. This conclusion is contrary to the usual assumption concerning stimulus variance, namely, that it is constant or that it increases with stimulus intensity.

If stimulus variance does not increase with stimulus intensity, then what is it related to? The results of this experiment suggested a hypothesis, to wit: Stimulus variance is less for stimulus distributions on that part of the sensory continuum where internal observations are most frequent. In other words, stimulus distributions are more compact and discriminability is greater in the region where internal observations occur more often. According to this hypothesis, all internal observations arising from all the different intensity levels determine stimulus variance. Let us see how this hypothesis accounts for differences in the slope of the ROC curves for different stimulus conditions and different stimulus pairs.

In the five-stimuli condition and in the eight-stimuli condition, the different stimuli are (within limits) presented equally of ten. Now consider the region of the sensory continuum on which the internal observations occur. The different stimulus distributions must overlap in the middle of this region. Hence, when all trials are combined, internal observations must be more frequent in the middle of the continuum than at the extremes. By our hypothesis, the variance of stimulus distributions in the middle of the continuum should be less than those at the extremes. Thus, this hypothesis is consistent with our conclusion that $\sigma_{\mathrm{C}}$ is less than $\sigma_{\mathrm{A}}$ and $\sigma_{\mathrm{E}}$.

Now consider the stimulus condition in which the weakest tone, $A$, is presented on $40 \%$ of the trials (five-stimuli-biased). This will increase the observations at the weaker end of the sensory continuum and decrease the observations elsewhere. Hence, by our hypothesis, $\sigma_{\mathrm{A}}$ should be smaller and $\sigma_{\mathrm{C}}$ and $\sigma_{\mathrm{E}}$ should be larger in the five-stimuli-biased condition than in the other two stimulus conditions. Consequently, $\sigma_{\mathrm{A}} / \sigma_{\mathrm{C}}$ should be smaller and, as we find (Table 2), the slope of the ROC curve for Stimuli A and C should be less when Stimulus A is more frequent. ${ }^{7}$

Finally, we should note that the data on discriminability do not support this hypothesis. If $\sigma_{\mathrm{C}}$ and $\sigma_{\mathrm{E}}$ are both larger in the five-stimuli-biased condition than in the other conditions, then Stimuli $\mathbf{C}$ and $\mathbf{E}$ should be less discriminable in that 
condition. The results are the opposite; $d_{\mathrm{e}}^{\prime}$ tends, if anything, to be larger in the five-stimuli-biased condition (Table 1).

Obviously, more direct tests can be made of the hypothesis that relates stimulus variance and the distribution of internal observations. Such tests seem necessary. It is the assumption that stimulus distributions are invariant from one experimental condition to another that gives SDT the power to relate performance in different situations. The limits of application of this assumption should be determined. It is tempting to explain some of the known limitations of SDT, namely, the effects of uncertainty of the signal frequency and of presentation frequency (as in the study of Markowitz \& Swets, 1967), in terms of this hypothesis.

The third aspect of the data we will examine does not raise questions about the assumptions of SDT. Rather, it concerns the way in which Os set criteria on the sensory continuum. When three or five categories are used, the location of the criteria are unchanged by changes in the presentation frequency of the stimulus. However, when nine categories are used, "weak" responses are less frequent in the condition in which weak stimuli are more frequent. This corresponds to a shift of the criteria toward the weaker end of the sensory continuum when weak stimuli are more frequent. This same shift has been found in previous work on category judgments (see, for instance, Stevens \& Galanter, 1957). This responsivity to the relative frequency of presentation of stimuli in the nine-category conditions, but not in the three- or five-category conditions, may be yet another instance of the importance of the "magic number" (Miller, 1956). It may be possible to place and hold two or even four criteria on the sensory continuum as a function of the range of stimuli. With eight criteria, this may be impossible.

\section{CONCLUSION}

The reasonably linear ROC curves suggest that SDT can be applied to tasks in which more than two stimulus values are presented and the $O$ uses a rating scale, i.e., to category judgment procedures. However, two findings of this study cast doubt on certain assumptions of SDT.

First, the dependence of discriminability upon the number of categories indicates once again that ROC curves obtained with different response conditions are not necessarily equivalent. The data support the interpretation that criterion variance increases with an increase in the number of criteria.

Second, for the less intense stimuli, the slope of the ROC curve is, under certain conditions, greater than unity. This finding calls into question the usual assumptions about the stimulus distributions, namely, that they are Gaussian with constant variance or variance that increases with an increase in stimulus intensity. Similarly, the assumptions that the distributions are Rayleigh or exponential are questioned by this finding. In fact, the data suggest that stimulus variance may depend upon the distribution of internal observations over all trials.

Obviously, this latter conclusion can be subjected to more direct experimental tests. Since background noise and feedback were not used in this experiment, the relevance of these results to the usual detection and recognition procedures remains open.

\section{REFERENCES}

EGAN, J.P., SCHULMAN, A.I., \& GREENBERG, G. Z. Operating characteristics determined by binary decisions and by ratings. Joumal of the Acoustical Society of America, 1959, 31, 768-773.

EMMERICH, D. S. ROCs obtained with two signal intensities presented in random order, and a comparison between yes-no and rating ROCs. Perception \& Psychophysics, 1968, 3, 35-40.

GREEN, D. M., \& SWETS, J. A. Signal detection theory and psychophysics. New York: Wiley, 1966.

MARKOWITZ, J., \& SWETS, J. A. Factors affecting the slope of empirical ROC curves: Comparison of binary and rating responses. Perception \& Psychophysics, 1967, 2, 91-100.

MILLER, G. A. The magical number seven, plus or minus two: Some limits on our capacity for processing information. Psychological Review, $1956,63,81-97$.

NACHMIAS, J. Effects of presentation probability and number of response alternatives on simple visual detection. Perception \& Psychoplysics, 1968, 3, 151-155.

STEVENS, S. S., \& GALANTER, E. H. Ratio scales and category scales for a dozen perceptual continua. Journal of Experimental Psychology, $1957,54,377-411$.

SWETS, J. A., \& SEWALL, S. T. Stimulus versus response uncertainty in recognition. Journal of the Acoustical Society of America, 1959, 31, 1126-1128.

SWETS, J. A., TANNER, W. P., \& BIRDSALL, T. G. Decision processes in perception. Psychological Review, 1961, 68, 301-340.

THURSTONE, L. L. A law of comparative judgment. Psychological Review, 1927, 34, 273-286.

TORGERSON, W. S. Theary and methods of scaling New York: Wiley, 1958.

WICKELGREN, W. A. Unidimensional strength theory and component analysis of noise in absolute and comparative judgments. Journal of Mathematical Psychology, 1968, 5, 102-122.

\section{NOTES}

1. This work was supported in part by NSF Grant GB 1462 to the University of Pennsylvania, and in part by NIH Grant MH 07990 to Eastern Pennsylvania Psychiatric Institute. I am grateful to Mrs. Sally Kraska for her extensive help in data collection and collation. The complete data in relative frequencies are available from the author

2. Address: Department of Psychology, University of Pennsylvania, Philadelphia, Pennsylvania 19100.

3. These assumptions are the same ones Torgerson (1958, Chap. 10) made in extending Thurstone's (1927) treatment of comparative judgments to category judgments. If we assume further that all criteria have the same distributions, we have what Torgerson calls Condition $B$ of the law of categorical judgment $(1958$, p. 208$)$.

4. Note that response proportions for the stronger stimuli are plotted on the ordinate. The additional assumption is sometimes made that the mean-to-sigma ratio, $\Delta m / \Delta \sigma \quad\left(\Delta m=\mu_{B}-\mu_{A}\right.$, $\Delta \sigma=\sigma_{\mathrm{B}}-\sigma_{\mathrm{A}}$ ), is constant. Taking $\Delta \mathrm{m} / \Delta_{\sigma}$ to be $4, R O C$ curves have been fitted to data with a single free parameter. See Green and Swets (1966, Chap. 4) for numerous instances of such fits.

5. Nachmias (1968), however, finds ROC curves to be unaffected by a priori probability of the signal for both rating-scale and binary-choice procedures when visual stimuli are used.

6 . In addition, assumptions about the underlying stimulus distribution could be called into question by such data In fact, category-judgment procedures such as the one used here offer the potential of separating the effect of criterion variance and the effect of the stimulus distribution. For instance, do curves for different stimulus pairs, obtained from the same block of trials, vary systematically (e.g., are the changes in slope the same) with specific criteria or with specific values of response proportions? The data presented here for $O S C$ is not orderly enough to answer such a question.

7. To be sure, this effect is found in only three of the four comparisons. The exception occurs when a nine-category scale is used. However, as we noted in the introduction, if criterion variance is large relative to stimulus variance, then stimulus variance has little effect on the slope of the ROC curve. As we concluded above, the criterion variance is largest with a nine-category scale. Note that we cannot predict changes in the slope for the $R O C$ curve for Stimuli $C$ and $E$. With an increase in the frequency of presentation of Stimulus $A$, both $\sigma_{\mathrm{C}}$ and $\sigma_{\mathrm{E}}$ are assumed to be larger, but we do not know the relative value of $\sigma_{C} / \sigma_{E}$ in the three conditions.

(Accepted for publication May 12, 1969.) 